During his tour, Sir John Russell visited more than forty experiment stations and inspected nearly ninety research schemes, in most cases discussing the work with the individual in charge. The posts under the Council's schemes are of temporary nature only and a plea is made for the provision of a number of permanent appointments, to be filled gradually by selected investigators of proved ability. By this means, specially experienced men would be available to deal with difficult problems at the various stations, where local resources were inadequate. Such a scheme would inevitably necessitate an increase in the financial grant made to the Council, but the return in enhanced efficiency would seem to justify this.

Much of the research in agricultural science in India is not so widely known as it deserves to be, due largely to the practice of publishing results in a number of small communications. The preparation of a series of monographs by competent persons is suggested, setting forth the results obtained by Indian workers and pointing out how they differ from those obtained elsewhere. Such publications would greatly raise the prestige of Indian research work and also prove of value to teachers and research workers throughout India itself.

\title{
Radio Transmission and the Ionosphere
}

$\mathrm{M}$ ARCONI'S discovery that radio waves followed the curvature of the earth indicated that the signals were reflected back to earth by an electrified condition of the upper atmosphere. In 1925 Prof. E. V. Appleton in England and Breit and Tuve of the Carnegie Institution (U.S.A.) gave definite experimental demonstrations of the existence of such a mirror.

Since these experiments were made, research on the electrified region of the atmosphere, called the ionosphere, has made great progress. It is now known that several distinct layers of ionization exist. The first, called the $E$ layer, is at a height of about sixty miles and is capable of reflecting fairly long waves. The second, called the $F_{1}$ layer, is capable of reflecting shorter waves, at a height of about 120 miles, and the third, the $F_{2}$ layer, reflects still shorter waves at a height of about 180 miles. The ability of these layers to reflect radio waves depends on the number of electrified particles present, either electrons or electrified air molecules. In an article communicated to the Radio Review of Australia of June by a member of the staff of the Carnegie Institution, it is described how the ionosphere affects radio transmission.

The author refers to the recent discovery by Dr. J. B. Dellinger, of the U.S. Bureau of Standards, of sudden fade-outs of high-frequency radio signals on the daylight side of the earth, and that these fade-outs were connected with the hydrogen prominences which sometimes appear on the sun. It is believed that all these fade-outs occur simultaneously with solar eruptions. When a fade-out occurs, no reflections are obtained from the $E, F$ or $F_{1}$ layers. It is just as if one were looking into a looking glass and suddenly the reflection disappeared. The cloud of electrons which prevent the waves being transmitted may be considered as a 'blanket' covering the entire daylight hemisphere of the earth at a height of $40-60$ miles.

A very pronounced fade-out occurred in April 1936 ; a sudden brightening was observed in the region around a spot on the sun. One minute later radio reflections from the ionosphere ceased and a large change also occurred in the earth's magnetism and in the earth currents. The heights of the reflecting layers recorded at the Carnegie Institution's observatories in Peru and Western Australia and by other organizations, serve to indicate the nature of the effects produced.
The improved quality of the ionosphere during times of sunspot activity makes a greater range of wave-lengths available for long-distance transmission. Unfortunately, times of sunspot maximum are most favourable for the occurrence of magnetic storms. These storms are believed to be due to swarms of ions and neutral corpuscles ejected from the sun with such speed that they reach the earth in about a day. Impinging on the atmosphere, these corpuscles give rise to auroral displays in high latitudes and seriously impair radio communication, when the path of the radio waves passes close to the radio zone--a region about $20^{\circ}$ from the geo-magnetic pole. Most of the radio traffic between the United States and Europe traverses such a path, with the result that on days of great magnetic disturbances those radio circuits are never available.

On the other hand, radio traffic between the United States and points in South America is not seriously impaired by magnetic disturbance. An ingenious application of this fact was made by one of the commercial companies during the severe magnetic disturbances on April 24-28, 1937. Radio messages intended for European points were 'routed' through the company's station in South America and retransmitted to Europe, with the result that communication was maintained throughout the disturbance.

Another interesting application of scientific knowledge concerns the scheduling of trans-Atlantic broadcast programmes. Research has shown that times of magnetic disturbances tend to recur at 27-day intervals, corresponding to the period of rotation of the sun. On this basis, it is possible to predict a month or two in advance the days that are likely to be unsatisfactory for long-distance transmission and the days that are likely to be satisfactory. International broadcasts, scheduled for pick-up by one of the large networks, are arranged so far as possible to come when no magnetic storms are expected.

The means by which magnetic storms affect radio transmission is not clearly understood. The effects of magnetic storms on radio transmission are markedly different from the effects produced by the ultraviolet light from solar prominences. While storm effects are most strongly manifested in high latitudes, the fade-outs are most pronounced near the equator. Furthermore, the fade-outs occur only during day. light hours, while the storms disturb radio transmission on both the day and the night sides of the earth. 